\title{
Higher Education Stakeholders' Views on Learning Analytics Policy Recommendations for Supporting Study Success
}

\author{
https://doi.org/10.3991/ijai.v1i1.10978 \\ Dirk Ifenthaler ( $\left.{ }^{\bowtie}\right)$, Jane Yau \\ University of Mannheim, Mannheim, Germany \\ dirk@ifenthaler.info
}

\begin{abstract}
Learning analytics show promise to support study success in higher education. Hence, they are increasingly adopted in higher education institutions. This study examines higher education experts' views on learning analytics utilisation to support study success. Our main research question was to investigate how ready higher education institutions are to adopt learning analytics. We derived policy recommendations from an international systematic review of the last five years of learning analytics research. Due to the lack of rigorous learning analytics research and adoption in Germany, this study focusses on the German university context and examines how ready German university stakeholders are to adopt learning analytics. In order to validate the policy recommendations, we conducted an interview study from June to August 2018 with 37 German higher education stakeholders. The majority of participants stated that their institutions required further resources in order to adopt learning analytics but were able to identify what these resources were in order for successful implementation.
\end{abstract}

Keywords-Learning analytics, higher education, adoption, study success, policy recommendation

\section{Introduction}

Learning analytics (LA) are increasingly adopted and utilised in higher education institutions in countries such as Australia, UK and the USA [1]. Ifenthaler [2] defines LA as the use of static and dynamic information about learners and learning environments, assessing, eliciting and analysing it, for real-time modelling, prediction and optimization of learning processes, learning environments, as well as educational decision-making. They are essential data-driven tools, which allow educators to view the learning progress of students so that they can be supported if they are underachieving or at risk. LA can also be used to motivate students to stay on their university courses and therefore facilitate and increase study success [3].

LA can be descriptive, predictive or prescriptive and offer different ways in which they can be designed, implemented and deployed to facilitate students' learning and their retention on courses [4-6]. LA data for summative reporting are obtained from 
sources such as course assessments, surveys, student information systems, learning management system activities, and forum interactions by descriptive analytics [7]. Similar data from those sources and attempts to measure onward learning success or failure are utilised by predictive analytics [6]. Algorithms to predict commonly the study success and whether students retain on their courses as well as suggesting immediate interventions are deployed by prescriptive analytics [8]. Typically, a student profile and their associated learning progress can be viewed, examined and appropriate alerts and/or actions can be taken [9]. The advantages of utilising LA in learning environments are a) increasing students' learning (experiences and effectiveness) and their learning motivation [10], and thereby, reducing student dropout or inactivity and increasing study completion [11], and b) providing personalised and adaptive learning paths via the specific goals set by the teacher or student to support the learning process [12]. However, the use of LA outside Australia, UK and the USA is still relatively rare $[4,13,14]$.

The aim of our current project 'Utilising Learning Analytics for Study Success' is to locate evidence in empirical studies published in scientific journals that the utilisation of LA does indeed increase study success and thereupon motivate institutions in other countries to adopt them to reduce student dropout. Further aims of the project include to development of a set of policies for higher education institutions to adopt LA capabilities into their existing organisational infrastructure and learning environment. In order to validate derived policy recommendation, this contribution focusses on the acceptance of the derived prevention and intervention LA tools to increase the study success by higher education experts. As part of this work and based on the systematic review, we derived policy recommendations on different levels of the higher education system (mega, macro, meso, micro level), which can potentially be used by stakeholders to successfully implement LA in their organisation. First, we present a literature review on LA implementation and related policy recommendations. Then, we present our qualitative study and related findings. Finally, we present conclusions, limitations and future work.

\section{$2 \quad$ Literature Review}

Study success includes the successful completion of a first degree in higher education to the largest extent, and the successful completion of individual learning tasks to the smallest extent [15]. As some of the more common and broader definitions of study success include terms such as retention, persistence, graduation rate and the opposing terms include withdrawal, dropout, non-completion, attrition and failure [16].

LA show promise to enhance study success in higher education [17]. For example, students often enter higher education academically unprepared and with unrealistic perceptions and expectations of academic competencies for their studies [18]. Both, the inability to cope with academic requirements as well as unrealistic perceptions and expectations of university life, in particular with regard to academic competencies, are important factors for leaving the institution prior to degree completion [16]. However, 
Sclater and Mullan [1] reported on the difficulty to isolate the influence of the use of LA, as often they are used in addition to wider initiatives to improve student retention and academic achievement.

Still, a number of reports currently exist in the area of LA including policy recommendations, each of which are detailing their individual policy recommendations for their geographical contexts. For example, Colvin, et al. [19] provided a set of policy recommendations for the Australian context:

- "Facilitating broader institutional, cross institutional and government discussions of LA and its capacity to inform sectorial challenges.

- Developing capacity building initiatives. This may manifest as professional development, secondments, and postgraduate course opportunities.

- Developing and supporting new models of education leadership that embrace complexity and enables innovation, organizational agility and adaptivity".

Five successful LA implementation-enabling factors in Australia include [19]:

- "Higher education leaders coordinate a high-level LA task force;

- Leverage existing national data and analytics strategies and frameworks;

- Establish guidelines for privacy and ethics;

- Promote a coordinated leadership programme to build institutional leadership capacity;

- Develop an open and shared analytics curriculum (to develop systematic capacity for LA by training skilled professionals and researchers)."

A similar set of policy recommendations was provided by Ferguson, et al. [4] in the European context, who also presented a discussion on how some countries such as Australia, Denmark, The Netherlands and Norway have successfully adopted LA. Table 1 presents a summary of the policy recommendations by Ferguson, et al. [4].

In Denmark, all schools began in 2016 a large-scale adoption of LA funded by the Ministry of Education. Similarly, in Norway, a research centre 'SLATE' on LA funded by the Ministry of Education was established and have three main focuses: Data sharing, vocabularies for activity descriptions, and privacy and best practice guidelines. In The Netherlands, two government-funded organisations are employed to integrate ICT for education including LA.

Cardinali, et al. [20] presented policy recommendations in three different sectors schools, higher education and workplace learning (see Table 2). 
Table 1. Summary of policy recommendations for the European context

\begin{tabular}{|c|c|}
\hline Focus area & Recommendations \\
\hline $\begin{array}{l}\text { Policy leadership and } \\
\text { governance practices }\end{array}$ & $\begin{array}{l}\text { Develop common visions of LA that address strategic objectives and priorities } \\
\text { Develop a roadmap for LA within Europe } \\
\text { Align LA work with different sectors of education } \\
\text { Develop frameworks that enable he development of analytics } \\
\text { Assign responsibility for the development of LA within Europe } \\
\text { Continuously work on reaching common understanding and developing new } \\
\text { priorities }\end{array}$ \\
\hline $\begin{array}{l}\text { Institutional leadership } \\
\text { and governance prac- } \\
\text { tices }\end{array}$ & $\begin{array}{l}\text { Create organisational structures to support the use of LA and help educational } \\
\text { leaders to implement these changes } \\
\text { Develop practices that are appropriate to different contexts } \\
\text { Develop and employ ethical standards, inclunding data protection }\end{array}$ \\
\hline $\begin{array}{l}\text { Collaboration and } \\
\text { networking }\end{array}$ & $\begin{array}{l}\text { Identify and build on work in related areas and other countries } \\
\text { Engage stakeholders throughout the process to create LA that have useful features } \\
\text { Support collaboration with commercial organisations }\end{array}$ \\
\hline $\begin{array}{l}\text { Teaching and learning } \\
\text { practices }\end{array}$ & $\begin{array}{l}\text { Develop LA that makes good use of pedagogy } \\
\text { Align analytics with assessment practices }\end{array}$ \\
\hline $\begin{array}{l}\text { Quality assessment and } \\
\text { assurance practices }\end{array}$ & $\begin{array}{l}\text { Develop a robust quality assurance process to ensure the validity and reliability of } \\
\text { tools } \\
\text { Develop evaluation checklists for LA tools }\end{array}$ \\
\hline Capacity building & $\begin{array}{l}\text { Identify skills required in different areas } \\
\text { Train and support researchers and developers to work in this field } \\
\text { Train and support educators to use analytics to support achievement }\end{array}$ \\
\hline Infrastructure & $\begin{array}{l}\text { Develop technologies that enable development of analytics } \\
\text { Adapt and employ interoperability standards }\end{array}$ \\
\hline
\end{tabular}

Table 2. Summary of policy recommendations for school, higher education and workplace learning

\begin{tabular}{|l|l|}
\hline \multicolumn{1}{|c|}{ Sector } & \multicolumn{1}{|c|}{ Recommendations } \\
\hline School & $\begin{array}{l}\text { Importance of democratic control } \\
\text { Data use considerations } \\
\text { Need for capacity-building } \\
\text { Focus on ethical questions }\end{array}$ \\
\hline Higher education & $\begin{array}{l}\text { Data standards } \\
\text { Identify requirements for data collection } \\
\text { Introduce standards for privacy, ethics \& data protection } \\
\text { Introduce and encourage the use of data standards } \\
\text { Ensure data is associated with metadata using standard conventions } \\
\text { Share standard datasets, with which others can be compared } \\
\text { Support for practitioners } \\
\text { Identify and share good practice } \\
\text { Enable networking and community support } \\
\text { Enable the sharing of expertise across countries \& disciplines } \\
\text { Support for institutions } \\
\text { Produce model agreements for institutions to use and adapt } \\
\text { Support institutions to evaluate the tools and resources on offer } \\
\text { Introduce procedures for due diligence when deploying interventions } \\
\text { Enable institutions to retain control of their data }\end{array}$ \\
\hline Workplace learning & $\begin{array}{l}\text { Map political context } \\
\text { Identify key stakeholders } \\
\text { Identify desired behaviour changes } \\
\text { Develop engagement strategy } \\
\text { Analyse internal capacity to effect change } \\
\text { Establish monitoring and learning frameworks }\end{array}$ \\
\hline
\end{tabular}


For the German context, our research group developed policy recommendations based on a systematic literature review and the particularities of the German higher education sector with a specific focus on study success [14]:

- Develop, support and sustain new models of education leadership that embrace the interdisciplinary nature of learning analytics requiring a combination of teams with skills from computer science, psychology, cognitive science, education and so on. Required attributes include innovation, adaptation to high-speed, high-paced changes to technology as well as excellent pedagogical skills for learning analytics development. Identify the skills required in different areas of learning analytics development. Train and support researchers and developers to work in these different areas.

- Develop practices that are appropriate and fitting to the needs of different institutions with different sizes of student groups, courses, staff numbers, etc. Train and support educators and staff to enhance achievement using learning analytics.

- Develop assessment practices, which are aligned with learning analytics in the institution.

- Develop organisational structures to support the use of learning analytics and help educational leaders to implement these changes.

- Develop a robust quality assurance process to ensure the validity and reliability of the tools as well as evaluation checklists.

- Develop capacity building initiatives, rewarding staff and institution(s) with certificates for professional development and providing training and postgraduate course opportunities. Engaging stakeholders in creating LA systems with useful features that support study success.

- Develop an open and shared learning analytics curriculum to increase the number of qualified professionals. Encourage the sharing and re-teaching and related skills.

- Continuation in the monitoring and adherence to ethics, data protection and privacy, which may infringe on individuals as well as the institution(s) involved.

Similarly, we derived the following set of policy recommendations for successful implementation within broader cross-institutional LA capacity building within Germany [14]:

- Enable institutional, cross institution and government discussions of learning analytics and their capacity to inform arising challenges from different perspectives: educational, administrative, financial, staff and technological constraints.

- Develop a national ethical board to stipulate that the ethics of each individual institution is met.

- Develop a learning analytics toolkit, which can be adaptive to the needs of individual institutions but is also transferrable for increased ease of use nationwide. Develop standardized technologies to enable learning analytics employment. 


\section{$3 \quad$ Research Questions and Methodology}

The current study aims to validate LA policy recommendations for the German higher education sector which were derived from the findings of a systematic review [14]. The overriding research question is as follows: Do experts of the German higher education sector confirm and accept policy recommendations for the implementation of LA for supporting study success in?

Our structured interview study was conducted from June 2018 to August 2018. We first collected a list of suitable participants; they had a level of expertise in educational technology and were a professional staff at a German higher educational institution. Some of these experts work directly or indirectly with LA and have different degrees of expertise of LA. The list was drawn from participants' list from German e-learning conferences and the Handbook of Mobile Learning in Germany [21]. Subsequently, we contacted them via email to request whether they were willing to participate in the interview study. The interview study was initiated by a pilot study of six experts from our university and these were conducted face-to-face. The pilot study confirmed the expected comprehension by experts and that the questions would be answered as intended and thus eliminating any ambiguity.

Participants in the main interview series included $N=31$ experts, which was conducted via remote conferencing due to practical reasons given the time and resources constraints as the participants were located in various parts of Germany. All of the interviews were recorded with the participants' consent and stored securely for later anonymous transcription and analysis. 10 out of 37 participants were female and the age range from 27 to 60 . Their areas of expertise include Information Management (with focus on workplace learning), Business Mathematics/Education, Educational Science, Computer/Data Science, Electronic/Mechanical Engineering, Psychology, and Web Technologies.

The interview was divided into eight sections - 1) Learning culture, 2) Study success, 3) Technology acceptance, 4) Understanding of LA, 5) Current LA projects (if any), 6) Strategies, policies and guidelines, 7) Time and resources, and 8) Demographic information.

The interview transcriptions were analysed using content data analysis and specifically we searched for evidence in the interview transcriptions to support/reject our policy recommendations (as presented above). Participants were also asked to provide their perspectives on the following topics: learning culture, study success, technology acceptance, understanding of LA, advantages and barriers to the adoption of LA as well as how ready their institution is to implement LA. The interviews were conducted in the German language and then transcribed and analysed.

The limitations of this study include the subjective opinions of each participant, which may not represent their institution truly. Two different researchers conducted the interviews due to practical reasons; this may also cause subjectivity by each researcher in the way the questions were posed. The researcher analysing the interview transcripts may also interpret the interviews subjectively according to his/her knowledge in this domain. 


\section{$4 \quad$ Results and Discussion}

\subsection{Learning culture, study success and technology acceptance}

Participants were asked to provide their opinions on what they understood as learning culture and study success. Various descriptions were provided on learning culture including: a) from the perspective of learners which was internal, and how they organise their learning to meet their learning goals and objectives, and b) from the perspective of the educational institution which was external and how different universities provide different forms of teaching, mentoring, seminars, supervision and so on. Learning culture could therefore in fact be a combination of both internal and external learning and teaching processes; and also it could be a combination of both selforganised and externally-organized processes. A positive learning culture could be one that motivate or encourage students to be active participants of their own learning, and be more engaging in order to obtain increased learning effectiveness and enjoyment of their studies. Learning culture could also mean the different methods of learning/teaching such as individual, or team-based, or based on different theoretical learning/teaching philosophies such as constructivist, or situated learning in different settings and communities. Learning culture could also relate to how learners learn in different scenarios or courses, or how they prepare for their exams, how often and for how long they spend on their studies. One participant differentiated and had the opinion that learning culture was very different to teaching culture and the teaching culture has direct influences on the learning culture. The participant argued that the lecturer could motivate and engage the learners with different methods and tools, and that there is a large range how learning cultures could look like between different subjects and departments across a university as well as between different universities. Therefore, it could be argued that the learning culture is decided by the educator who determines the types of coursework being assigned (micro perspective) [22]. Another perspective given by another participant of learning culture is actually "what should be learnt", "who should learn it", and "how should it be learnt", "how should the learning process be", and "what should not be learnt".

One participant provided a broader and longer-term description of study success such as the ability to accomplish one's goals in finishing studies and obtaining a good job and ensuring future financial security [23]. Similarly, another participant argued that study success implied being able to find a job and carry out the job requirement and specifications; the job itself would be different for everyone since people have different interests. Other participants provided narrower descriptions such as if the student could pass a course or degree at the university [15]. Another perspective is that study success can be increased if a learner studies a subject that is suited to their personality, passion or interest. Otherwise, it may be much more difficult to meet the demands of the course and produce successful learning outcomes. Therefore, study success should also not just be directly related to examination, but learning and applying knowledge relevant for lifelong learning [16]. Strategies for study success suggested by participants include the use of and mixture of different methods of teaching/learning. Students should not just be consumers of information but producers of 
knowledge. An overall description as seen by many participants' answers includes the completion of their studies as well as being able to secure and carry out a job adequately thereafter.

Some participants provided broad opinions regarding students' and staff view on technology acceptance including that it requires trust, which can sometimes be difficult to gain if there is a strong disposition against the use of technology for learning [24]. Some participants maintained that technology acceptance is not normally an issue since they are based in a Computer Science department. For some other students, they may be more willing to adopt technology if they aid them with their learning processes or "help them learn faster in the course". One educator explained that in his/her course some technologies currently being used (the platform for sharing learning materials and recorded lectures) are well accepted by the students. In this case, the students "are usually more willing to use tools and existing products/software to achieve than to code something from scratch themselves. They prefer to use additional technologies, because it facilitates and makes their learning process more efficient and effective". He summarized technology acceptance to include four deciding factors: performance, expectations, social influences, and facilitating condition. Another participant saw that some students were unwilling to use the technology due to the concern that traces of data were left for example they did not want to be known how often they were accessing the learning platform [25].

A participant who was a Computer Science professor stated that technology acceptance is extremely high in his department (the technologies included Communication and Internet technologies). He added that technologies must be user-friendly and have a high-quality user-interface in order for it to be accepted. Indeed, trust was an overarching element throughout the different expert interviews which was required in order for technologies to be accepted and then subsequently for LA to be adopted. In order to gain the trust, one participant identified that the technologies must be able to show the quality of the mentoring and feedback capabilities and system's processes should be transparent and ethical. Therefore, it is important to show, rather than hide, how the backend LA algorithms work. Many participants concluded that simplicity of the usage of the LA system was also an important requirement leading to successful implementation. One of the first issues/barriers relating to successful implementation is data protection, which has been a mentioned concern by almost every participant. One participant mentioned that in his Computer Science department, most of the staff trusted the use of digitalisation of teaching, but not yet LA.

Throughout the interview with different participants, data privacy and protection was a concern for the students [26]. Often, this was due to a misconception and lack of real understanding of what exactly happens to the data and who has access to it. Once the students would be explained exactly about what data was collected and how they were used, many of the participants agreed that they would be much more willing and enthusiastic about the usage of LA to enhance their study success [27-29]. 


\subsection{Understanding of LA, current LA projects, barriers to LA adoption}

Most participants could provide an accurate description of what LA constitutes. The following elaborations of a potential definition/description of LA was provided by participants:

"If you collect enough data, one can probably observe patterns of some things that can be improved. It is a type of data analysis, where one can see some practices, which relate to better results of the students in the end or some practices, which may lead to poorer results. Maybe one can also observe when students have more difficulties with their courses and when they are struggling more with one course more than another. This provides another way to know how the learners are coping in the courses in addition to the normal teaching/learning processes where there is minimum interaction. So one can identify which of the used teaching practices lead to either better or worse results for the students."

"By implementing a detailed learning analytics scheme, one is attempting to ask the students if they have any difficulties relating to different aspects of their university courses. One might indicate factors that one can make positive teaching/learning impact on and make it easier for the students to still learn everything that they are required to learn. So, I'm not talking about lowering the criteria for the students so it would be easier for them to pass, but to identify factors that make it more difficult for them to learn materials or to focus on their studies and try to do something about those factors. So LA is about retaining or increasing the teaching quality and at the same time enabling more students to finish their degree."

Due to the novelty of LA in German higher education institutions, there is limited research, or resources dedicated for the implementation of LA systems at universities in Germany. For example, one participant who came to work in Germany from overseas experienced a number of difficulties concerning data protection when attempting to implement a LA system. Most of the mentioned barriers to LA adoption were mostly financial constraints including personnel costs (sufficient and qualified multidisciplinary staff required to operate the different parts of the LA system, for example, pedagogical staff concerning the learning materials, data protection staff concerning data privacy aspects, IT staff concerning technical implementation and maintenance) and actual software, server and licensing costs for the implementation of the LA system [30]. Most participants mentioned that there were not any LA projects currently operating at their institution. Some LA projects currently running in the institution are listed below.

- LAPS project at Hochschule der Medien, Stuttgart - data mining methods are used to determine study success and risk dimensions of students.

- LeAP project integrated into the ILIAS learning management system at the University of Mannheim - currently, foundational research in LA but the research has advanced significantly and has overcome some obstacles including the technical constraints of data protection and how to connect different university systems together to facilitate a LA system/platform.

- FAMULUS at LMU Munich - Students learn through adaptive simulations and feedback in medicine including the use of LA. 
In summary, most participants agreed and emphasised that the first, large obstacle to LA implementation was data protection. In Germany, the rights to data protection are very important. For example, students have the rights not to provide directly information on how many hours they spent studying, or indirectly via traces of data left when logging on and off a learning management system. Another obstacle is the workload this creates on members of staff. A concern specified by a participant being "The more data one collects, the better it would be for the learning analytics. However, it might imply possible administering several surveys and questionnaires during the course and may conflict with the dynamics of the course and some teaching staff may not be willing to do so easily." Whilst these two points are seen as obstacles, one participant views it rather as a difficulty that can be navigated and overcome. The difficulties lie also on different levels for example one level is linked strongly with trust which according to the participant who is a computer scientist emphasized that a) they may not trust as much with other knowing their data as staff/students from students from other disciplines purely because they may have a better understanding about what one can do with the data, and b) trust requires a very social component which some computer scientists do not like/are not social. In order to overcome these issues, some strategies can be put in place such as reinstating that not all students' private data will be collected, only those relevant required and with the students' consent. There are also technical aspects, which include the connection of different systems inclusive of data protection issues, which require technical expertise of IT staff and can be problematic [9].

\subsection{Readiness to adopt $\mathrm{LA}$ and validation of policy recommendations}

We examined the responses to the interview question "How ready is your institution to adopt LA?". Six participants expressed that their institution was ready to adopt LA because their institution currently has LA research projects and possibly a system in place and they may effectively adopt more projects or implement LA in students' existing courses in a relatively straight-forward manner. It is also the case that some of these participants stated that they also have currently the personnel required including a professor, a postdoc and doctoral students in this area of research.

The majority of participants $(N=30)$ expressed that there are currently resources required by their institution before they can go ahead and adopt LA. In general, these participants expressed that their institution is mentally ready to adopt LA as the benefits of study success outweigh the costs [31]. The required resources include staff and technological capabilities [30].

Currently, many German higher educational institutions do not have the resources required to adopt LA, which is therefore a barrier to successful implementation. For example, several participants noted that their institutions are not very familiar with what LA entails and do not currently have any strategies for adoption. However, they are open to it and want to invest in it because they have the opinion that learners' data can enrich their learning processes. Some institutions are currently adopting digitalisation of teaching but not yet LA. One participant mentioned that some parts of their technical systems are ready for LA incorporation (but not all parts) and some data can 
also be made easily available (but not all the data). A further requirement includes that teachers and students need to be interested in the aspects of improving the teaching/learning processes via the use of data and LA.

Several participants emphasised the problem that there is a lack of learner's personal data relating to their learning processes, exam grades and so on, which makes predictions in Germany very difficult. Due to the strict data protection laws, this is not allowed and therefore eliminates/decreases the ability for LA systems to make accurate predictions based on students' data. The participant could be understand why the data cannot be made available especially given that the data can be anonymised.

Only one participant stated that he rejects most technologies including mobile learning devices and learning analytics and would prefer to use paper-based teaching and learning materials. Therefore, this participant stated that in his/her opinion, his/her institution should not be ready to adopt LA.

A further analysis of responses focussed on the validation of the policy recommendations for the German higher education sector. Table 3 shows each of the policy recommendation and the requirements for adoption of LA.

Table 3. Validation of policy recommendations for the German higher education sector

\begin{tabular}{|l|l|}
\hline \multicolumn{1}{|c|}{ Policy recommendation } & \multicolumn{1}{c|}{ Requirements for adoption } \\
\hline 1. Develop education leadership & Train and support researchers \\
\hline 2. Develop individualised practices & Train and support staff \\
\hline 3. Develop assessment practices & Train and support staff \\
\hline 4. Develop organisational structures & $\begin{array}{l}\text { Providing supportive structures, e.g., study consulting, } \\
\text { workshops }\end{array}$ \\
\hline 5. Develop a quality assurance process & Train and support staff \\
\hline 6. Develop capacity building initiatives & Train and support staff \\
\hline 7. Develop a learning analytics curriculum & Train and support staff \\
\hline 8. Adherence to data protection & $\begin{array}{l}\text { Become familiar and navigate } \\
\text { carefully with the guidelines, e.g., EU GDPR }\end{array}$ \\
\hline 9. Enable country-wide discussions & Train and support staff \\
\hline 10. Develop a national ethical board & Train and support staff \\
\hline $\begin{array}{l}\text { 11. Develop a standardised learning analytics } \\
\text { system }\end{array}$ & Train and support staff \\
\hline
\end{tabular}

One participant stressed the importance of LA leadership and role models because LA is still a very new field [32]. Experimental 'playgrounds' are required to understand, discuss, debate, test out all LA ideas and put them into practice and learn from these good/bad experiences and studies. It is also very important so that LA stakeholders understand fully what LA and personalised teaching entails. Insights into the advantages of adopting LA into their institution include

- Personalised teaching

- Individualised feedback

These two items are one participant's opinion of the best possible elements of teaching. Other mentioned advantages include 
- Saving time by exam corrections

- Finding out students' challenges with different aspects of the courses

- Improving the quality of the courses.

The main identified disadvantages is data privacy [29]. One participant used the example that often many people are willing to allow stores to have their personal data for marketing purposes for exchange of discounts. However, they may not be willing to allow educational institutions to access and store similar data, which can provide them with better current educational opportunities, which will lead to a better future for them. The general focus is to have the stakeholders fully understand what and how data is being used in order for them to accept the requirement of LA system in exchange for personalised teaching as well as individualised feedback leading to potential increased study success. The disadvantage is therefore the loss of some data privacy which is a limitation of LA system, however, when data privacy and protection and exactly what and how data is used is explained upfront, then some participants' experiences were that stakeholders found it easier to accept [25]. Asking students for information that they are not willing to share is also a disadvantage of the usage of LA. One solution is to anonymise the data although this may not be straight-forward. Currently, many HEIs in Germany are in the transitional process and some first implementation challenges regarding data have already been solved. Other strategies/solutions include attempting to make the users not feel 'watched/monitored' [27].

It is interesting to note that many of the participants have similar ideas about the advantages and disadvantages of LA implementation and ways to overcome the specific challenges. This implies that current challenges in successful LA implementation are widely known within the German education and research community and a list of protocol can be put together to accommodate the requirements and desires German HEIs have in order to adopt LA.

\section{Conclusion and Future Work}

The implications how to support stakeholders at higher education institutions in utilising LA to support study success are still under-documented. Remaining questions are for example: Will students be able to respond positively and proactively when informed that their learning progress is hindered or inactivated?; Will instructors be able to influence the at-risk students positively so that they will re-engage with the studies? In addition, ethical dimensions regarding descriptive, predictive and prescriptive LA need to be addressed with further empirical studies and linked to study success indicators $[25,27]$.

\section{Acknowledgement}

The authors acknowledge the financial support by the Federal Ministry of Education and Research of Germany (BMBF, project number 16DHL1038). 


\section{$7 \quad$ References}

[1] N. Sclater and J. Mullan, Learning analytics and student success - assessing the evidence. Bristol: JISC, 2017.

[2] D. Ifenthaler, "Learning analytics," in The SAGE encyclopedia of educational technology, vol. 2, J. M. Spector, Ed. Thousand Oaks, CA: Sage, 2015, pp. 447-451. https://doi.org/10. $\underline{1108 / \mathrm{rr}-08-2015-0202}$

[3] D.-K. Mah and D. Ifenthaler, "Students' perceptions toward academic comptencies: the case of German first-year students," Issues in Educational Research, vol. 28, no. 1, pp. 120-137, 2018.

[4] R. Ferguson et al., "Research evidence on the use of learning analytics - Implications for education policy," in "Joint Research Centre Science for Policy Report," 2016, [Online]. Available: http://publications.jrc.ec.europa.eu/repository/bitstream/JRC104031/lfna28294enn.pdf.

[5] D.-K. Mah, J. Y.-K. Yau, and D. Ifenthaler, "Future directions on learning analytics to enhance study success," in Utilizing learning analytics to support study success, D. Ifenthaler, J. Y.-K. Yau, and D.-K. Mah, Eds. Cham: Springer, 2019, pp. 313-321. https://doi.org/10.1007/978-3-319-64792-0 17

[6] D. Glick, A. Cohen, E. Festinger, D. Xu, Q. Li, and M. Warschauer, "Predicting success, preventing failure," in Utilizing learning analytics to support study success, D. Ifenthaler, D.-K. Mah, and J. Y.-K. Yau, Eds. Cham: Springer, 2019, pp. 249-273. https://doi.org/ 10.1007/978-3-319-64792-0_14

[7] N. Arthars, M. Dollinger, L. Vigentini, D. Y. Liu, E. Kondo, and D. M. King, "Empowering teachers to personalize learning support," in Utilizing learning analytics to support study success, D. Ifenthaler, D.-K. Mah, and J. Y.-K. Yau, Eds. Cham: Springer, 2019, pp. 223-248. https://doi.org/10.31234/osf.io/3xu5t

[8] R. S. Baker and G. Siemens, "Educational data mining and learning analytics," in The Cambridge handbook of the learning sciences, R. K. Sawyer, Ed. 2 ed. Cambridge, UK: Cambridge University Press, 2015, pp. 253-272. https://doi.org/10.1017/cbo9781139519 $\underline{526.016}$

[9] D. Klasen and D. Ifenthaler, "Implementing learning analytics into existing higher education legacy systems," in Utilizing learning analytics to support study success, D. Ifenthaler, J. Y.-K. Yau, and D.-K. Mah, Eds. New York, NY: Springer, 2019, pp. 61-72. https://doi.org/10.1007/978-3-319-64792-0 4

[10] C. Schumacher and D. Ifenthaler, "Why learning analytics need to care for motivational dispositions of students," presented at the AERA Anual Meeting, New York, NY, USA, 13-04-2018, 2018.

[11] K. E. K. Chai and D. C. Gibson, "Predicting the risk of attrition for undergraduate students with time based modelling," in Proceedings of cognition and exploratory learning in the digital age, D. G. Sampson, J. M. Spector, D. Ifenthaler, and P. Isaias, Eds. Maynooth, Ireland: IADIS Press, 2015, pp. 109-116.

[12] K. Fuchs, P. A. Henning, and M. Hartmann, "INTUITEL and the hypercube model - Developing adaptive learning environments," Journal on Systemics, Cybernetics and Informatics, vol. 14, no. 3, pp. 7-11, 2016.

[13] N. Sclater, A. Peasgood, and J. Mullan, Learning analytics in higher education: A review of UK and international practice. Bristol: JISC, 2016.

[14] D. Ifenthaler, D.-K. Mah, and J. Y.-K. Yau, "Utilising learning analytics for study success. Reflections on current empirical findings," in Utilizing learning analytics to support study 
success, D. Ifenthaler, J. Y.-K. Yau, and D.-K. Mah, Eds. New York, NY: Springer, 2019, pp. 27-36. https://doi.org/10.1007/978-3-319-64792-0 2

[15] C. S. Sarrico, "Completion and retention in higher education," in Encyclopedia of international higher education systems and institutions, T. P. and S. J., Eds. Dordrecht: Springer, 2018. https://doi.org/10.1007/978-94-017-9553-1 87-1

[16] D.-K. Mah, "Learning analytics and digital badges: potential impact on student retention in higher education," Technology, Knowledge and Learning, vol. 21, no. 3, pp. 285-305, 2016. https://doi.org/10.1007/s10758-016-9286-8

[17] M. D. Pistilli and K. E. Arnold, "Purdue Signals: Mining real-time academic data to enhance student success," About campus: Enriching the student learning experience, vol. 15, no. 3, pp. 22-24, 2010. https://doi.org/10.1002/abc.20025

[18] D.-K. Mah and D. Ifenthaler, "Academic staff perspectives on first-year students' academic competencies," Journal of Applied Research in Higher Education, vol. 9, no. 4, pp. 630640, 2017. https://doi.org/10.1108/jarhe-03-2017-0023

[19] C. Colvin et al., Student retention and learning analytics: A snapshot of Australian practices and a framework for advancement. Canberra, ACT: Australian Government Office for Learning and Teaching, 2015.

[20] F. Cardinali et al., "Policy recommendations for learning analytics from three stakeholder workshops," in "Learning Analytics Community Exchange," 2015, [Online]. Available: http://www.laceproject.eu/publications/policy-recommendations-lace-workshops.pdf.

[21] C. Gloerfeld and C. de Witt, Eds. "Handbuch Mobile Learning." Heidelberg: Springer, 2018.

[22] S. Pöntinen, P. Dillon, and P. Väisänen, "Student teachers' discourse about digital technologies and transitions between formal and informal learning contexts," Education and Information Technologies, vol. 22, no. 1, pp. 317-335, 2017. https://doi.org/10.1007/s10639$\underline{015-9450-0}$

[23] V. Tinto, "Reflections on student retention and persistence: moving to a theory of institutional action on behalf of student success," Studies in Learning, Evaluation, Innovation and Developmental Psychology, vol. 2, no. 3, pp. 89-97, 2005.

[24] N. Nistor, A. Göğüş, and T. Lerche, "Educational technology acceptance across national and professional cultures: a European study," Educational Technology Research and Development, vol. 61, no. 4, pp. 733-749, 2013. https://doi.org/10.1007/s11423-013-9292-7

[25] D. Ifenthaler and C. Schumacher, "Student perceptions of privacy principles for learning analytics," Education Tech Research Dev, vol. 64, no. 5, pp. 923-938, 2016. https://doi.org/10.1007/s11423-016-9477-y

[26] D. Ifenthaler and M. W. Tracey, "Exploring the relationship of ethics and privacy in learning analytics and design: implications for the field of educational technology," Education Tech Research Dev, vol. 64, no. 5, pp. 877-880, 2016. https://doi.org/10.1007/s11423016-9480-3

[27] D. West, H. Huijser, and D. Heath, "Putting an ethical lens on learning analytics," Educational Technology Research and Development, vol. 64, no. 5, pp. 903-922, 2016. https://doi.org/10.1007/s11423-016-9464-3

[28] S. Slade and P. Prinsloo, "Learning analytics: Ethical issues and dilemmas," American Behavioral Scientist, vol. 57, no. 10, pp. 1510-1529, 2013. https://doi.org/10.1177/000276 $\underline{4213479366}$

[29] D. Ifenthaler and C. Schumacher, "Releasing personal information within learning analytics systems," in Learning technologies for transforming teaching, learning and assessment at large scale, D. G. Sampson, J. M. Spector, D. Ifenthaler, P. Isaias, and S. Sergis, Eds. New York, NY: Springer, 2019, pp. 3-18. https://doi.org/10.1007/978-3-030-15130-0 1 
[30] D. Ifenthaler, "Are higher education institutions prepared for learning analytics?," TechTrends, vol. 61, no. 4, pp. 366-371, 2017. https://doi.org/10.1007/s11528-016-0154-0

[31] D. West, D. Heath, and H. Huijser, "Let's talk learning analytics: A framework for implementation in relation to student retention," Online Learning, vol. 20, no. 2, pp. 1-21, 2016. https://doi.org/10.24059/olj.v20i2.792

[32] S. Buckingham Shum and T. A. McKay, "Architecting for learning analytics. Innovating for sustainable impact," EDUCAUSE Review, vol. 53, no. 2, pp. 25-37, 2018.

\section{Authors}

Dirk Ifenthaler Dirk Ifenthaler is Professor and Chair of Learning, Design and Technology at the University of Mannheim, Germany and UNESCO Deputy Chair of Data Science in Higher Education Learning and Teaching at Curtin University, Australia. His research focuses on the intersection of cognitive psychology, educational technology, data analytics and organisational learning (www.ifenthaler.info).

Jane Yin-Kim Yau is a Researcher in Learning Analytics \& Mobile Learning at the Chair for Learning, Design and Technology at the University of Mannheim, Germany.

Article submitted 2019-06-03. Resubmitted 2019-07-19. Final acceptance 2019-07-19. Final version published as submitted by the authors 